\title{
Physics and causation*
}

\author{
Michael Esfeld, University of Lausanne, Department of Philosophy \\ Michael-Andreas.Esfeld@unil.ch \\ (forthcoming in Foundations of Physics, \\ special issue in honour of the $80^{\text {th }}$ birthday of Peter Mittelstaedt)
}

\begin{abstract}
The paper makes a case for there being causation in the form of causal properties or causal structures in the domain of fundamental physics. That case is built in the first place on an interpretation of quantum theory in terms of state reductions so that there really are both entangled states and classical properties, GRW being the most elaborate physical proposal for such an interpretation. I then argue that the interpretation that goes back to Everett can also be read in a causal manner, the splitting of the world being conceivable as a causal process. Finally, I mention that the way in which general relativity theory conceives the metrical field opens up the way for a causal conception of the metrical properties as well.
\end{abstract}

Keywords: causal properties, classical properties, direction of time, dispositions, entanglement, Everett, GRW, measurement problem, metaphysics of science, metrical field, physical structures, propensities, quantum mechanics, spontaneous localizations, state reductions

\section{Introduction}

Peter Mittelstaedt and I taught a course together at the University of Cologne in the winter term 2001/02, that course being centred on philosophical theories of causation. He repeatedly asked me what the physical foundations of causation were, and I was unable to answer that question at the time. In this paper, I would like to sketch out the way in which I tend to answer this question today (without expecting Peter Mittelstaedt to agree with that answer). I endorse a realist view of causation, claiming that causation is a fundamental physical feature, more precisely that the fundamental physical properties or structures are causal properties or structures. This is a minority view, being opposed to the mainstream view rooted in empiricism according to which causation is not a fundamental feature of the world.

The latter view has been forcefully set out by Bertrand Russell (1912) in a famous paper in which he claims that a realist attitude to causation is not compatible with modern physics. In the contemporary discussion, notably John Norton (2007a and b) has put forward such a view. Of course, Russell, Norton and others are right in claiming that there is no question of an $a$ priori principle of causation to be imposed upon physics. A case for causation in physics can only be made by considering the interpretation of the fundamental physical theories (see also the discussion between Frisch 2009a, 2009b and Norton 2009). In this vein, I shall go into the interpretation of quantum physics (section 2), explaining how the view of causation as a

I am grateful to Paul Busch for helpful comments on the draft of this paper, to Mauro Dorato for discussions and common work related to GRW and causation, and to an anonymous referee for his or her suggestions. 
fundamental physical feature fits well into the interpretation of quantum physics in terms of state reductions, GRW being the most prominent example of such an interpretation (section 3). Nonetheless, this view is also compatible with the Everett interpretation (section 4). Finally, I will briefly mention the interpretation of general relativity (section 5) and in conclusion sum up the arguments for taking causation to be anchored in physics (section 6).

\section{2. $\quad$ The measurement problem in quantum physics}

Quantum theory is one of the two fundamental physical theories, general relativity theory being the other one. Notably the application of quantum theory to cosmology and the search for a quantum theory of gravity, which sets out to unify quantum field theory and general relativity theory, make clear that quantum theory is not a theory about measurement - that is, not a theory about the behaviour of microphysical objects relative to measurement devices -, but a candidate for a fundamental and universal theory of nature. The universal character of quantum theory is pointed out by Peter Mittelstaedt (1998) in his by now classical book on the interpretation of quantum mechanics and the measurement process. A fundamental theory is one whose laws and explanations do not depend on any other theories, and a universal theory is one whose laws apply to everything that there is in the world. Thus, for instance, statistical mechanics is not a fundamental theory, since its laws and explanations depend on laws and explanations that apply to single systems.

The upshot of these developments is that in today's philosophy of science, it is clear that quantum theory as such does not yield any reason to abandon scientific realism. Notably following the debate about Bell's theorem and the subsequent experiments, it is evident that quantum theory forces us to change our vision of nature, that is, our Naturphilosophie, but that as such it does not throw the presupposition that science tells us something about the constitution of nature into question (for an assessment, see especially the papers in Cushing and McMullin 1989 and Redhead 1995, in particular chapter 3).

Nonetheless, the standard textbook presentation of quantum mechanics still accords measurement a prominent role, whereas, for instance, in the standard textbook presentation of general relativity theory, no such stress is laid on measurement. The standard textbook presentation of quantum mechanics, going back to von Neumann (1932, chapter VI), suggests that when a measurement occurs, the dynamics of quantum systems as described by the Schrödinger equation is no longer valid and one has to switch to another dynamics, which simply consists in the postulate that when a measurement happens, the measured quantum system adopts a definite numerical value of the measured property. The reason for this sudden switch to another dynamics is that according to the Schrödinger dynamics, whenever one system interacts with another system, and be it the interaction of a microscopic quantum system with a macroscopic measurement device, the states of the two systems become entangled. However, definite numerical values are observed as measurement outcomes, and a measurement apparatus is in a state in which it indicates a definite numerical value if and only if the measured quantum system is in a state in which it possesses a definite numerical value of the measured property.

Although not unmotivated, von Neumann's proposal is completely ad hoc. Measurement processes and devices are not natural kinds, but scientists employ various physical systems as measurement devices if they suit their interests. It is not possible to give a precise physical definition of a measurement process and a measurement apparatus, since there is no physical 
difference that distinguishes a measurement process from other physical interactions. Measurement devices are an invention of human beings that occurs late in cosmic evolution and that presupposes the existence of macroscopic systems that are not subject to quantum entanglement. Taking cosmology into account, it is evident that there have been processes of the dissolution of quantum entanglement (state reductions) in cosmic evolution independently of humans for humans to be able to use certain physical systems as measurement devices. Such processes were the basis on which classical physical systems such as molecules, organisms and finally humans developed. The measurement problem thus is not about measurement in particular. It is a placeholder for the general problem how to understand the transition from quantum systems in entangled states to systems that possess classical properties. It is this problem that the thought experiment of Schrödinger's cat highlights: one can countenance atoms being in superposed and entangled states, but not cats.

This is a real problem for both physicists and philosophers concerned with the metaphysics of science, since there is no established physical fact that one can cite as a solution to this problem. This lack of an established physical fact also is the reason why the standard textbook presentation of quantum mechanics still resorts to the unsatisfactory dualism of two dynamics that goes back to von Neumann. There has of course been physical progress since the days of von Neumann, but no definitive physical solution to the measurement problem. Decoherence is such an established physical fact, but it changes nothing as regards quantum entanglement: applied to measurement, the state of the quantum object and the measurement apparatus still is an entangled one (an improper mixture, to use the term introduced by d'Espagnat 1971, chapter 6.3), and not a product state (a proper mixture) (see e.g. Adler 2003 and Schlosshauer 2004). It is obvious why decoherence cannot dissolve entanglement, since decoherence takes place entirely within the Schrödinger dynamics. Decoherence at most shows why entanglement is not accessible to a local observer, but cannot provide for its reduction.

\section{GRW: the idea of a unified dynamics, and causation}

This situation is the motivation for trying to do better than von Neumann did. There is evidence that there is quantum entanglement, as highlighted by the Bell-type experiments, and there is evidence that there are classical properties. The ontologically most conservative solution to the measurement problem consists in searching for a unified dynamics that does justice to both entanglement in the quantum domain and to classical properties when it comes to macroscopic objects. Such a dynamics, however, can be achieved only by amending the Schrödinger dynamics, more precisely by breaking its linearity.

The only elaborate physical proposal for a unified dynamics that accepts both the existence of quantum entanglement and provides for the transition to classical properties is the one going back to Ghirardi, Rimini and Weber (1986) (GRW) (forerunners of this proposal include notably Pearle 1976 and Gisin 1984). GRW add a stochastic term to the linear Schrödinger equation such that for an isolated, non-massless microscopic quantum system whose wave function (state vector) has a certain spatial spread that stochastic term indicates a very low probability for spontaneous localization - that is, for a state reduction that ends up in a spontaneous adoption of a rather definite position. It will on average take $10^{16}$ seconds for such an isolated system to undergo a spontaneous localization. By contrast, when one considers a macroscopic system that is composed of a large number of microscopic quantum 
systems, such a system will adopt a definite position in an extremely short time. Due to entanglement, any spontaneous localization of one of the microscopic systems composing a macroscopic system implies the spontaneous localization of all the others and thus of the macroscopic system as a whole (see Ghirardi 2005, chapters 16.8 and 17, for an elaborate presentation). It is of course not the task of a philosopher to assess the physical quality of the proposal of GRW. I consider this proposal since it is hitherto the only elaborate physical theory of a dynamics of state reductions.

The stochastic term that GRW add to the Schrödinger equation and that results in an indeterministic dynamics is not a hidden variable. What GRW set out to do is to integrate von Neumann's state reduction postulate in the Schrödinger dynamics in order to achieve a unified dynamics. The spontaneous localizations occur independently of measurement processes. Measurement simply is one interaction among others that does not call for a special treatment in the GRW version of quantum mechanics. GRW thereby is able to account for the cosmic evolution that leads from quantum systems to classical systems and finally organisms, including humans and their technological inventions. The gain thus is unification: provided that one grants that both the quantum and the classical domain exist, GRW set out to tell us, in contrast to von Neumann's postulate, how both these domains hang together.

However, to be precise, the GRW amendment to the Schrödinger equation does not result in a microscopic quantum system adopting exactly one definite numerical value of position (apart from the mathematical fact that, in classical as well as in quantum mechanics, position has a continuous spectrum of values so that its value can never be exactly one number). The stochastic term that GRW add to the Schrödinger equation amounts to the wave function being centred around a peak as a result of a spontaneous localization of the system. But that peak is not a point, and, moreover, the wave function is not zero outside that peak. This fact is known as the problem of the tails of Schrödinger's cat (the tails problem for short).

According to the standard solution to this problem that goes back to Albert and Loewer (1996), the requirement of there being precise numerical values (in other words, the eigenstate-eigenvalue link) has to be abandoned. Thus, the price that we have to pay for this solution - and hence for having a unified dynamics at our disposal - is that the notion of microscopic localization becomes somewhat vague and that, in general, we have to countenance values that are objectively there, but slightly vague or fuzzy. (The discussion on the tails objection focused in recent years on the counting anomaly raised by P. Lewis 1997 and Clifton and Monton 1999; but the reply to that objection by Bassi and Ghirardi 1999 and 2001 seems to me to be entirely convincing; see also Wallace 2008, end of section 3).

Although the original proposal of GRW looks like an ontology of particles, quantum systems being particles each of which has a certain probability of undergoing a process of spontaneous localization, GRW are not committed to an ontology of particles. In contrast to Bohm's quantum theory, there is nothing like particles possessing always a definite position and thus a definite trajectory in GRW. There are processes of spontaneous localization, but these are processes of state reduction of superposed or entangled states, and quantum systems in these latter states do not possess a definite localization and hence no definite trajectory. Since there is interaction, the states that are to be considered as the basis for spontaneous localizations are not superposed states of isolated quantum systems, but entangled states that involve a lot of quantum systems. 
Quantum entanglement is the main motivation for a position that is known as ontic structural realism and that is one of the main currents in contemporary metaphysics of science, going beyond an ontology of particles (see notably Ladyman 1998, French and Ladyman 2003, Esfeld 2004). A physical structure can be conceived as a network of concrete physical relations - such as the relations of quantum entanglement - among objects that are nothing but what stands in these relations. There is no need for structural realism to claim that there are no objects at all and thus no need to change standard first order logic, which quantifies over objects (see the so-called moderate structural realism of Esfeld and Lam 2008 in contrast to French and Ladyman 2003; but see also the more moderate formulations in Ladyman and Ross 2007, chapters 2 to 5). The point is that these objects do not possess any identity independently of the relations in which they stand. There hence are no intrinsic properties on which these relations could supervene, and the objects cannot be individual particles.

Let us assume that ontic structural realism is a metaphysical framework that fits quantum entanglement, pointing out the appropriate way in which quantum entanglement goes beyond an ontology of particles. GRW have the means to take quantum entanglement, thus conceived, into account. Nothing hinders one to conceptually develop GRW in such a way that the disposition for spontaneous localization is regarded as being inherent to the entangled state as such, instead of being an intrinsic property of individual quantum particles. In other words, the probability for an entangled state to undergo a state reduction in the form of the spontaneous localization of the objects that stand in the relations of entanglement in question depends on the number of objects that the entangled state includes. The result of this interpretative move is exactly what GRW claim, namely that a macroscopic system consisting of a great number of quantum systems that are entangled with each other adopts a rather precise position in physical space in the form of a spontaneous localization of these quantum systems in an extremely short time, whereas in the case of there being only a few quantum systems that are entangled with each other, the probability for the whole system to adopt a precise position in physical space is very low. Hence, in developing the ontology of GRW, one can waive a commitment to quantum systems being individual particles.

That move paves the way for extending GRW to quantum field theory, including quantum gravity, as well. There are of course non-local correlations in GRW, including correlations between space-like separated events of spontaneous localization, but there is no non-local interaction. GRW violate the condition of outcome independence in the derivation of the Bell inequalities, but not the condition of parameter independence. (Mittelstaedt 2000 takes GRW to explain these non-local correlations in terms of the process resulting in spontaneous localization being their common cause).

Furthermore, following Bell (1987), one can conceive GRW in such a way that the reduction of entangled states results in flashes centred around space-time points. Thus conceived, GRW are not committed to there being a globally privileged reference frame or coordinate system, as has been shown by Tumulka (2006) (see also Maudlin 2008 for a discussion). The flash version of GRW hence is compatible with special as well as general relativity. It can be set out in such a manner that, for instance, the question of the temporal order of the two measurements in a Bell-type experiment on a pair of spin 1/2 systems in the singlet state separated by a space-like interval has no significance at all. Although the work of Tumulka (2006) is only a first step, there is thus reason to hope that it will eventually turn out 
to be possible to develop an interpretation of quantum theory that acknowledges processes of state reduction existing in nature without having to maintain that there is a globally privileged reference frame or coordinate system, imposing an objective temporal order on space-like separated events.

Taking state reductions seriously, as do GRW, has important ontological consequences, and it is here that causation comes in. According to GRW, definite numerical values of statedependent properties exist, but only as a result of the dissolution of entanglement, that is, as a result of state reductions in the form of spontaneous localizations. The state reductions lead to one definite numerical value existing at the exclusion of the other possible definite numerical values. An entangled state therefore is best conceived as the disposition to bring about definite numerical values of state-dependent properties through state-reduction - in other words, the disposition for spontaneous localization. That disposition is itself a real and actual property, not a mere potency.

Treating entanglement as a disposition that is a real and actual property fits into what is known as the causal theory of properties (see notably Shoemaker 1980 and Bird 2007). According to this theory, insofar as properties are certain qualities, they are powers to bring about certain effects. Thus, insofar as entangled states are certain real and actual, qualitative physical states, they are the power to bring about definite numerical values through spontaneous localization - or, in short, the power to produce spontaneous localizations. In contrast to macroscopic dispositions such as the disposition of water to dissolve sugar, the power that entangled quantum states are does not depend on outside triggering conditions for its manifestation. That is the point of the disposition being one for spontaneous localization.

There is no need for causal properties to be intrinsic properties of individual particles. Nothing hinders one to apply the causal theory of properties to physical structures such as the structures of entanglement: insofar as entanglement is a network of concrete physical relations, that network is the power to bring about spontaneous localizations such that there are definite numerical values that are correlated in a specific manner (see Esfeld 2009 for details). Hence, as regards entanglement, there are only possible definite numerical values each of which can become actual at the exclusion of the other ones. However, this is not a mere potency, but a really existing power to bring about such values (for more details about quantum dispositions in GRW and the arguments in the following four paragraphs, see Dorato and Esfeld forthcoming).

Introducing dispositions and thus causation in this manner in the interpretation of quantum physics yields a clear answer to the question what the properties of quantum objects are if there are no properties with definite numerical values. Suggesting that there are no properties at all if there are no definite numerical values does not make sense, since a physical system cannot exist without possessing physical properties, and properties with definite numerical values cannot develop out of nothing. And saying that there simply is the wave function or state vector begs the question, since the wave function or state vector is a mathematical tool to represent physical reality, but not itself physical reality. In other words, one has to spell out an account of what the physical reality as represented by the wave function or state vector is. Conceiving the ontology of GRW in terms of dispositions provides for such an account: the properties that there are in entangled states when there are no definite values are dispositions to develop such values, and the causal theory of properties makes clear how such dispositions can be real and actual properties. 
Conceiving entangled states as dispositions in the form of causal powers implies a further commitment: taking probabilities into account, we have to say that the powers that the entangled states are amount to propensities, that is, dispositions that have a certain quantifiable strength for spontaneous manifestation in a certain manner, that is, producing a certain numerical value at the exclusion of other values. That strength is expressed in the form of probabilities. In other words, in this framework, we are committed to applying the propensity theory of probabilities to the quantum probabilities. The advantage of doing so is that we get the account of probabilities that is needed for the quantum probabilities, namely objective single case probabilities. Quantum probabilities clearly apply to single cases, and they are objective probabilities, concerning features of the quantum domain itself, instead of our knowledge. The propensity view of probabilities meets these demands, since it takes probabilities to be anchored in dispositions that have a certain quantifiable strength to manifest themselves spontaneously in a certain manner.

Frigg and Hoefer (2007), by contrast, regard the GRW probabilities as fitting into a Humean theory of probabilities as well. However, a Humean theory of probabilities, such as the one of David Lewis (1994), has to resort to the parameters of simplicity and strength and the best balance between them. But these are epistemic parameters. It is therefore doubtful whether such a theory can really yield objective, single case probabilities. Mittelstaedt (1997, in particular pp. 168-170) does not countenance the propensity interpretation of quantum probabilities either, although he endorses an individualistic interpretation of quantum mechanics by contrast to an ensemble interpretation. However, in this case, it seems logical to apply probabilities to single cases as well, and the only clear way of doing so is by conceiving them as propensities. The propensity interpretation of quantum probabilities has been refined considerably since it had first been introduced notably by Popper (1959) (see in particular the papers of Suárez 2004a, 2004b and 2007 as well as Dorato 2007).

Finally, there is a further feature that an interpretation of quantum theory that acknowledges state reductions has to include and that becomes intelligible in the framework of the causal theory of properties. Amending the Schrödinger equation with a stochastic term as GRW do in order to account for state reductions has not only the consequence that the dynamics is indeterministic, but also that it is not time-reversal invariant. In other words, the GRW equation is a candidate for a fundamental law of nature that is not time-reversal invariant. Quantum systems that have undergone a spontaneous localization may of course again enter into entangled states, but conceiving a process that runs from a spontaneous localization back to the entangled state that existed before that localization would contradict the GRW law, more precisely the stochastic term that GRW add to the Schrödinger equation. Again, that feature of GRW has an important advantage: it provides for an explanation of the direction of time. As Albert (2000, chapter 7) has shown, the GRW processes of spontaneous localization are not only irreversible, thus singling out a direction of time, but they can also serve as the origin of all time-asymmetric phenomena. Conceiving entangled states in terms of dispositions (causal powers) leads to an explanation of this lack of time-reversal invariance: the production of an effect by a cause is the paradigmatic example of an irreversible process. Consequently, if spontaneous localization is the manifestation of a disposition, it is evident why processes of state reduction can in principle not be reversed and why they are the foundation of the direction of time. In other words, if causation is a feature of the domain of fundamental physics, there being causal properties in that domain, then the 
dynamics describing that domain cannot be time-reversal invariant, since the production of an effect by a cause is not a time-reversal invariant process.

To sum up, there are arguments that are independent of the issue of causation in fundamental physics and that speak in favour of searching for a unified dynamics in the interpretation of quantum physics, as do GRW. The main argument is that in doing so, one does justice to both the existence of entanglement in the quantum domain and to the existence of classical properties when it comes to macroscopic objects. Within the framework of a unified dynamics, nothing hinders one from maintaining that quantum theory provides for a complete description of the quantum domain - in other words, the wave function (state vector) is such a complete description, and there is no need to supplement this description with the assumption of so-called hidden variables (as is done in Bohm's theory of the quantum domain, which in addition to the description by the wave function assumes that quantum systems are individual particles, possessing always a definite numerical value of position and thus a definite trajectory in space-time). Furthermore, in acknowledging the real existence of classical properties, there then is no reason to commit oneself to an inflationary ontology of the world splitting itself up in infinitely many branches of the universe as a result of measurements (or simply as a result of decoherence), since no state reductions are admitted (as according to the interpretation going back to Everett 1957). Having thus based an interpretation of quantum physics in terms of a unified dynamics on arguments that are independent of the issue of causation in physics, the ontological commitments of that interpretation then lead us, as spelled out in this section, to acknowledging causation in the form of dispositions and thus causal properties as a fundamental physical feature.

\section{The Everett interpretation and causation}

Although the interpretation of quantum physics that admits state reductions is the locus classicus for anchoring dispositions and thus causation in the domain of fundamental physics, making a case for causation in quantum physics is not necessarily tied to an interpretation of quantum physics in terms of entangled states being dispositions for developing into classical properties. Causation can be situated within the other two main contenders in the interpretation of quantum physics as well. As regards Bohm's theory, it seems possible to propose a causal reading of the quantum potential, it being a causal structure that moves the particles around. Let us, however, consider the Everett interpretation rather than Bohm's interpretation in a little more detail, since it enables us to further pursue the issue of causation in fundamental physics.

The Everett interpretation takes the Schrödinger dynamics to be the complete dynamics of quantum systems. It does not recognize state reductions, and it does hence not recognize the real existence of classical properties. One is in this case committed to regarding the quantum structures of entanglement as being universal: they encompass all the objects in the world and all their dynamical properties, including all the macroscopic objects and in the last resort also the consciousness of observers (the last consequence has first been highlighted by Albert and Loewer 1988 and Lockwood 1989, chapters 12 and 13, in the so called many minds interpretation of quantum theory). According to the way in which this interpretation is usually set out, the world splits into infinitely many branches that exist in parallel so that all the objects in the world including the consciousness of each observer are infinitely many times duplicated, existing in infinitely many branches of the universe, and having one of the 
possible dynamical values of their properties in each of these branches. In other words, all the possible definite numerical values of a property that enter into the superpositions and entanglement do in fact exist, but distributed among infinitely many branches of the universe.

The observation of classical properties is usually accounted for in this framework in the following manner: the structures of entanglement develop into a process that is known as decoherence. Decoherence is a process leading in a very short time to the different terms of an entangled state no longer interfering with each other. A local observer who stands within such a structure of entanglement therefore has in her observations no access to the other terms of the superposition, which exist in other branches of the universe. In a nutshell, the world appears classically to us, because, being local observers, we cannot observe the structures of entanglement as a whole (which nevertheless really exist) (see Wallace 2008, section 4, for a detailed assessment of the contemporary discussion about the interpretation of quantum theory that goes back to Everett).

It is in this interpretative framework also possible to consider the quantum structures of entanglement as causal structures. This interpretation has to answer the following question: What does the physical reality of the quantum structures of entanglement consist in, so that these structures can develop through decoherence into a splitting of the world in infinitely many branches, which do not interfere with each other and which contain definite numerical values of quantum properties each? A clear answer to this question that recognizes the physical reality of the structures of quantum entanglement without confusing that physical reality with the mathematical reality of the state vector (wave function) consists in saying the following: the quantum structures of entanglement are causal structures, being the power or disposition to produce through decoherence infinitely many branches of the universe, which do not interfere with one another. On this reading, decoherence is a causal process, consisting in the manifestation of causal structures in the form of the structures of entanglement. In order to vindicate this reading, one would have, however, to show how decoherence can be conceived as an irreversible process, although decoherence is situated entirely within the Schrödinger dynamics.

Let us pursue the crucial point one step further: one cannot content oneself with the claim that one should take the quantum wave function (state vector) seriously, although that claim is of course well taken. The quantum state vector is a mathematical structure, living in configuration space and representing physical reality, but it is not itself physical reality. In an interpretation of a physical theory, one therefore has to answer the question what the physical reality as represented by a mathematical structure (such as the wave function or state vector in configuration space) amounts to. The answer to this question cannot consist in saying that what is physically real is defined by its position in fourdimensional space-time, since entangled states do not possess a definite localization and are independent of spatio-temporal distances. One obtains a forceful answer to this question by employing a causal criterion of reality: real physical structures distinguish themselves from mathematical structures in that they are causally efficacious. In other words, something is a candidate for a real physical entity in contrast to a mere mathematical one if and only if it can be conceived as being a causal power, that is, as being a disposition to produce certain effects. That criterion applies to the interpretation of quantum physics independently of whether or not one recognizes state reductions. 
Since quantum entanglement is independent of spatio-temporal distance, the structures of entanglement can be regarded as being more fundamental than the metrical structures of classical space-time. Indeed, important research in quantum gravity takes the direction of treating classical space-time as not being fundamental (see e.g. Kiefer 2004, notably chapter 10). But what then is fundamental and how does what is physically fundamental distinguish itself from a mere mathematical entity? Again, one can represent the quantum reality in an abstract and general manner in terms of algebraic structures (see notably the algebraic representation of quantum field theory, in particular Haag 1992). But algebraic structures are a tool to represent physical reality so that one still has to answer the question of what that physical reality thus represented consists in and how real physical structures distinguish themselves from their representation in terms of mathematical structures. In early modern philosophy of nature, Leibniz objects to Descartes that the notion of spatio-temporal extension is not fundamental and that instead the causal notion of force is more fundamental. Leibniz hence defends a causal conception of matter. That conception still is the only alternative available to the definition of physical reality in terms of localization in space-time. The question of what the fundamental reality as represented by mathematical structures consists in hence leads to a general and powerful argument for taking causation in the form of causal structures to be a fundamental physical feature.

\section{5. $\quad$ Space-time and causation}

Let us briefly consider space-time itself as treated by general relativity theory. In classical physics up to and including special relativity, space-time is conceived as a passive background structure in which material objects and their properties are embedded. However, general relativity theory abandons this conception of space-time. According to general relativity theory, the metrical field contains itself energy, namely the gravitational energy. Hence, general relativity theory excludes a dualism between space-time as a passive background arena and matter as that what is inserted into this arena. Space-time, as constituted by the metrical field, is itself a dynamical entity and interacts with nongravitational energy-matter as well as with itself.

Since the metrical field contains the gravitational energy, it can be conceived as a material entity on a par with all the other physical fields. In this vein, one can regard gravitation as a fundamental physical interaction on a par with the other fundamental physical interactions the fact that gravitation is universal and includes all physical objects notwithstanding (see in particular Rovelli 2007, section 4). On this view, in short, the fact that space-time is no passive background structure in general relativity signifies that the spatio-temporal, gravitational structures are material structures as well. On this basis, it is therefore possible to conceive the spatio-temporal, gravitational relations as a causal structure as well: the metrical structures are the power or disposition to produce the gravitational effects (see Bartels 1996, pp. 37-38, Bartels 2009 and Bird 2009, section 2.3; see Livanios 2008 against this position however, simply pointing out the fact that the metrical structures are geometrical structures begs the question, since they contain the gravitational energy).

One may envisage going even one step further: the programme known as causal set theory provides for a precise mathematical model of how causal processes can build up space-time itself (see in particular Sorkin 1991 and Reid 2001). One can receive this programme as taking up Leibniz' characterization of matter in terms of points of force and employing this 
characterization in order to understand space-time itself: according to causal set theory, causal elements locally produce further causal elements, and these processes taken together constitute space-time (see Butterfield 2007, pp. 858-861, and Earman 2008, sections 7 and 8, for a philosophical assessment). However, the view of the metrical structures of space-time being causal structures is not committed to the programme of causal set theory.

\section{Conclusion}

In sum, we can say the following: nothing hinders one to conceive the fundamental physical structures as causal structures. By contrast to what the tradition going back to Russell (1912) claims, it is not only possible to interpret today's fundamental physical theories in a causal manner, but there are a number of arguments for doing so: general philosophical arguments from the metaphysics of properties (see e.g. Shoemaker 1980 and Bird 2007), the requirement to be able to distinguish real physical from mere mathematical structures, and several concrete arguments in the interpretation of quantum theory and the interpretation of general relativity theory.

These arguments drive us to an anti-empiricist ontology that rehabilitates the notions of causation and causal properties in the domain of fundamental physics: we have to countenance irreducible dispositions and consequently causal properties in the fundamental physical realm, and at least if we do so in the framework of an interpretation of quantum theory that admits state reductions, we are thereby also committed to objective, single case probabilities in the form of propensities, and we get a fundamental law that is time-reversal invariant, thus designating a direction of time. There is as such nothing wrong with these commitments. The tradition of dogmatic empiricism in the philosophy of science has been overcome since the 1960s, and there are sound arguments available for each of these ontological commitments, as indicated in this paper. These commitments bring fundamental science closer to the special sciences such as biology as well as to common sense - at least in the sense that the special sciences and common sense trade in dispositions, adopting a realist attitude towards causation, and that the phenomena they consider are nearly all not timereversal invariant. But again, this is a welcome result, since fundamental science, the special sciences and common sense are all about one and the same world. Note that the relative closeness to the special sciences and to common sense is a result, and not a starting point or an argument for the claims defended in this paper.

The renewed interest in metaphysics in contemporary philosophy started with descriptive metaphysics in the late 1950s (see e.g. Strawson 1959), which limited itself to describing the ontological commitments of common sense, refusing to engage in the project of revising these commitments. Today, a current known as metaphysics of science belongs to the mainstream in philosophy of science, seeking to build a vision of nature on the basis of our best scientific theories, thereby linking up with the German tradition of Naturphilosophie. Metaphysics of science clearly engages in a revision of the ontological commitments of common sense on the basis of science. But it would certainly not be wrong-headed if this current could link up with descriptive metaphysics in retaining some of its central commitments - such as the one to dispositions and causation -, insofar as there are independent arguments available for these commitments based on science. 


\section{Bibliography}

Adler, Stephen L. (2003): "Why decoherence has not solved the measurement problem: a response to P. W. Anderson”. Studies in History and Philosophy of Modern Physics 34B, pp. 135-142.

Albert, David Z. (2000): Time and chance. Cambridge (Massachusetts): Harvard University Press.

Albert, David Z. and Loewer, Barry (1988): “Interpreting the many worlds interpretation”. Synthese 77, pp. 195213.

Albert, David Z. and Loewer, Barry (1996): “Tails of Schrödinger's cat”. In: R. K. Clifton (ed.): Perspectives on quantum reality. Dordrecht: Kluwer. Pp. 81-91.

Bartels, Andreas (1996): "Modern essentialism and the problem of individuation of spacetime points". Erkenntnis 45, pp. 25-43.

Bartels, Andreas (2009): “Dispositionen in Raumzeit-Theorien”. In: C. F. Gethmann (ed.): Lebenswelt und Wissenschaft. XXI. Deutscher Kongress für Philosophie. Kolloquien. Hamburg: Meiner.

Bassi, Angelo and Ghirardi, Gian Carlo (1999): "More about dynamical reduction and the enumeration principle”. British Journal for the Philosophy of Science 50, pp. 719-734.

Bassi, Angelo and Ghirardi, Gian Carlo (2001): "Counting marbles: Reply to Clifton and Monton". British Journal for the Philosophy of Science 52, pp. 125-130.

Bell, John S. (1987): “Are there quantum jumps?” In: C. W. Kilmister (ed.): Schrödinger. Centenary celebration of a polymath. Cambridge: Cambridge University Press, pp. 41-52. Reprinted in J. S. Bell (1987): Speakable and unspeakable in quantum mechanics. Cambridge: Cambridge University Press, pp. 201-212.

Bird, Alexander (2007): Nature's metaphysics. Laws and properties. Oxford: Oxford University Press.

Bird, Alexander (2009): "Structural properties revisited". In: T. Handfield (ed.): Dispositions and causes. Oxford: Oxford University Press. Pp. 215-241.

Butterfield, Jeremy N. (2007): "Stochastic Einstein locality revisited". British Journal for the Philosophy of Science 58, pp. 805-867.

Clifton, Robert K. and Monton, Bradley (1999): "Losing your marbles in wave function collapse theories". British Journal for the Philosophy of Science 50, pp. 697-717.

Cushing, James T. and McMullin, Ernan (eds.) (1989): Philosophical consequences of quantum theory. Reflections on Bell's theorem. Notre Dame: University of Notre Dame Press.

d'Espagnat, Bernard (1971): Conceptual foundations of quantum mechanics. Menlo Park: Benjamin.

Dorato, Mauro (2007): “Dispositions, relational properties, and the quantum world”. In: M. Kistler and B. Gnassounou (eds.): Dispositions and causal powers. Aldershot: Ashgate. Pp. 249-270.

Dorato, Mauro and Esfeld, Michael (forthcoming): "GRW as an ontology of dispositions". Forthcoming in Studies in History and Philosophy of Modern Physics. Paper available at http://philsciarchive.pitt.edu/archive/00004870/

Earman, John (2008): "Reassessing the prospects for a growing block model of the universe". International Studies in the Philosophy of Science 22, pp. 135-164.

Esfeld, Michael (2004): "Quantum entanglement and a metaphysics of relations". Studies in History and Philosophy of Modern Physics 35B, pp. 601-617.

Esfeld, Michael (2009): “The modal nature of structures in ontic structural realism”. International Studies in the Philosophy of Science 23, pp. 179-194.

Esfeld, Michael and Lam, Vincent (2008): “Moderate structural realism about space-time”. Synthese 160, pp. 2746.

Everett, Hugh (1957): “'Relative state' formulation of quantum mechanics”. Reviews of Modern Physics 29, pp. 454-462. Reprinted in B. S. DeWitt and N. Graham (eds.) (1973): The many-worlds interpretation of quantum mechanics. Princeton: Princeton University Press. Pp. 141-149.

Field, Hartry H. (2003): "Causation in a physical world”. In: M. Loux and D. Zimmerman (eds.): The Oxford handbook of metaphysics. Oxford: Oxford University Press. Pp. 435-460.

French, Steven and Ladyman, James (2003): "Remodelling structural realism: quantum physics and the metaphysics of structure". Synthese 136, pp. 31-56. 
Frigg, Roman and Hoefer, Carl (2007): "Probability in GRW theory". Studies in History and Philosophy of Modern Physics 38B, pp. 371-389.

Frisch, Mathias (2009a): “'The most sacred tenet?' Causal reasoning in physics”. British Journal for the Philosophy of Science 60, pp. 459-474.

Frisch, Mathias (2009b): "Causality and dispersion: A reply to John Norton". British Journal for the Philosophy of Science 60, pp. 487-495.

Ghirardi, Gian Carlo (2005): Sneaking a look at God's cards. Unraveling the mysteries of quantum mechanics. Translated by Gerald Malsbary. Princeton: Princeton University Press.

Ghirardi, Gian Carlo, Rimini, Alberto and Weber, Tullio (1986): "Unified dynamics for microscopic and macroscopic systems". Physical Review D 34, pp. 470-491.

Gisin, Nicolas (1984): “Quantum measurements and stochastic processes”. Physical Review Letters 52, pp. 1657-1660 (and reply p. 1776).

Haag, Rudolf (1992): Local quantum physics. Berlin: Springer.

Kiefer, Claus (2004): Quantum gravity. Oxford: Oxford University Press.

Ladyman, James (1998): "What is structural realism?" Studies in History and Philosophy of Modern Science 29, pp. 409-424.

Ladyman, James and Ross, Don with Spurrett, David and Collier, John (2007): Every thing must go. Metaphysics naturalised. Oxford: Oxford University Press.

Lewis, David (1994): "Humean supervenience debugged". Mind 103, pp. 473-490. Reprinted in D. Lewis (1999): Papers in metaphysics and epistemology. Cambridge: Cambridge University Press. Pp. 224-247.

Lewis, Peter (1997): "Quantum mechanics, orthogonality, and counting". British Journal for the Philosophy of Science 48, pp. 313-328.

Livanios, Vassilios (2008): "Bird and the dispositional essentialist account of spatiotemporal relations". Journal for General Philosophy of Science 39, pp. 383-394.

Lockwood, Michael (1989): Mind, brain and the quantum. The compound 'I'. Oxford: Blackwell.

Maudlin, Tim (2008): "Non-local correlations in quantum theory: some ways the trick might be done". In: Q. Smith and W. L. Craig (eds.): Einstein, relativity, and absolute simultaneity. London: Routledge. Pp. 186209.

Mittelstaedt, Peter (1997): “Is quantum mechanics a probabilistic theory?” In: R. S. Cohen, M. A. Horne and J. Stachel (eds.): Potentiality, entanglement and passion-at-a-distance. Quantum mechanical studies for Abner Shimony. Volume 2. Dordrecht: Kluwer. Pp. 159-175.

Mittelstaedt, Peter (1998): The interpretation of quantum mechanics and the measurement process. Cambridge: Cambridge University Press.

Mittelstaedt, Peter (2000): “The problem of decoherence and the EPR-paradox". In: P. Blanchard, D. Guilini, E. Joos, C. Kiefer and I.-O. Stamatescu (eds.): Decoherence: Theoretical, experimental, and conceptual problems. Heidelberg: Springer. Pp. 149-159.

Norton, John (2007a): "Causation as folk science". In: H. Price and R. Corry (eds.): Causation, physics, and the constitution of reality. Russell's republic revisited. Oxford: Oxford University Press. Pp. 11-44.

Norton, John (2007b): "Do the causal principles of modern physics contradict causal anti-fundamentalism?" In: P. Machamer and G. Wolters (eds.): Thinking about causes: From Greek philosophy to modern physics. Pittsburgh: University of Pittsburgh Press. Pp. 222-234.

Norton, John (2009): "Is there an independent principle of causality in physics?" British Journal for the Philosophy of Science 60, pp. 475-486.

Pearle, Philip (1976): "Reduction of statevector by a nonlinear Schrödinger equation”. Physical Review D 13, pp. $857-868$.

Popper, Karl R. (1959): "The propensity interpretation of probability". British Journal for the Philosophy of Science 10, pp. 25-43.

Redhead, Michael L. G. (1995): From physics to metaphysics. The Tarner lectures. Cambridge: Cambridge University Press. 
Reid, D. D. (2001): “Introduction to causal sets: An alternative view of spacetime structure”. Canadian Journal of Physics 79, pp. 1-16.

Rovelli, Carlo (2007): “Quantum gravity”. In: J. N. Butterfield and J. Earman (eds.): Handbook of the philosophy of science. Philosophy of physics. Part B. Amsterdam: Elsevier. Pp. 1287-1329.

Russell, Bertrand (1912): “On the notion of cause”. Proceedings of the Aristotelian Society 13, pp. 1-26.

Schlosshauer, Maximilian (2004): "Decoherence, the measurement problem, and interpretations of quantum mechanics". Reviews of Modern Physics 76, pp. 1267-1305.

Shoemaker, Sydney (1980): “Causality and properties”. In: P. van Inwagen (ed.): Time and cause. Dordrecht: Reidel. Pp. 109-135. Reprinted in S. Shoemaker (1984): Identity, cause, and mind. Philosophical essays. Cambridge: Cambridge University Press. Pp. 206-233.

Sorkin, Rafael D. (1991): "Spacetime and causal sets”. In: J. C. d'Olivo, E. Nahmad-Achar, M. Rosenbaum, M. P. Ryan, L. F. Urrutia and F. Zertuche (eds.): Relativity and gravitation: classical and quantum. Singapore: World Scientific. Pp. 150-173.

Strawson, Peter F. (1959): Individuals. An essay in descriptive metaphysics. London: Routledge.

Suárez, Mauricio (2004a): “On quantum propensities: two arguments revisited”. Erkenntnis 61, pp. 1-16.

Suárez, Mauricio (2004b): "Quantum selections, propensities and the problem of measurement”. British Journal for the Philosophy of Science 55, pp. 219-255.

Suárez, Mauricio (2007): “Quantum propensities”. Studies in History and Philosophy of Modern Physics 38B, pp. 418-438.

Tumulka, Roderich (2006): “A relativistic version of the Ghirardi-Rimini-Weber model”. Journal of Statistical Physics 125, pp. 821-840.

von Neumann, Johann (1932): Mathematische Grundlagen der Quantenmechanik. Berlin: Springer. English translation Mathematical foundations of quantum mechanics. Translated by R. T. Beyer. Princeton: Princeton University Press 1955.

Wallace, David (2008): “The interpretation of quantum mechanics". In: D. Rickles (ed.): The Ashgate companion to the new philosophy of physics. Aldershot: Ashgate. 